\title{
Vibration Control Studies Using an Impedance Method
}

\author{
G.M. Kamath ${ }^{a}$, P. Senthil Kumar ${ }^{b}$ and M.V. Shivaprasad ${ }^{c}$ \\ ${ }^{a}$ (Corresponding author), Scientist, Advanced Composites Unit, National Aerospace Laboratories, \\ Bangalore, Email: gmkamath@css.cmmacs.ernet.in \\ ${ }^{b}$ Scientist, Advanced Composites Unit, National Aerospace Laboratories, Bangalore \\ ${ }^{c}$ Project Trainee, Advanced Composites Unit, National Aerospace Laboratories, Bangalore
}

\begin{abstract}
This paper presents a vibration control methodology that is useful in practical applications where the system to be controlled is difficult to model due to the presence of uncertainties or complex boundary conditions. The impedance control method uses a power flow approach wherein the controller is designed such that power flow into the structure is minimised. This is accomplished by using the dereverberated point impedance function at the actuator location for the design of the controller. The method is implemented and simulated for a cantilever beam with piezoceramic actuators. As a preliminary step towards real-time implementation of impedance control a simple state feedback algorithm is implemented using a dSPACE digital signal processor (DSP) card.
\end{abstract}

Keywords: vibration control, impedance method, dereverberated transfer function, piezoelectric materials

\section{INTRODUCTION}

Vibration control of structures has received wide attention for many years now, especially with the advent of smart materials, on account of its well-known benefits. This paper presents a control methodology that has many advantages for practical and realistic applications. The impedance control method is based on electrical circuit analogy that for maximum power dissipation in the circuit, the load impedance should be equal to the complex conjugate of the source impedance. This power flow approach has been exploited for acoustic and noise control ${ }^{1,2}$, and for vibration control ${ }^{3,4,5}$.

Impedance control method minimises the power flow into the structure coming from external disturbances and from the controller itself. Thus, for vibration reduction, this power flow into the structure is negative. This is done by considering the dereverberated point mobility transfer function between the collocated actuator and sensor. The mobility and its inverse, the impedance, are considered because the product of the input and the output of these transfer functions is the power flow. A dereverberated transfer function (DTF), as opposed to the reverberated transfer function (RTF), is devoid of the poles and zeros that one sees in the latter. The poles and zeros in the RTF are caused due to the reflection of the traveling waves at the boundaries of the structure and their interference (constructive and destructive) with transmitted waves. Thus, the DTF could be construed as a transfer function between the actuator and the sensor on a structure that is infinitely large. The DTF thus models only the local dynamics at the actuator/sensor location. This has an advantage in that it improves the robustness of the controller because the uncertainties in the structure away from the actuator/sensor location do not affect the controller. Moreover, this can be obtained easily from the RTF obtained using analytical or experimental data. The latter option is especially useful in complex structures where analytical methods are inaccurate or cumbersome. Also, since the DTF does not consider the poles and zeros of the structure, it offers a more broadband control than other conventional control methods such as rate feedback.

The DTF is obtained from the RTF through different approximate methods. The need for approximate methods arises due to the fact that the exact DTF obtained from the RTF is non-causal, and hence cannot be implemented in practice. One method of obtaining the DTF is through the cepstrum of the impulse response ${ }^{3}$. Another method is by taking the 
logarithmic average of the $\mathrm{RTF}^{6}$. The third method is by critically damping the poles and zeros of the $\mathrm{RTF}^{5}$. This paper uses the latter method on account of its simplicity and ease of application in practical situations.

This paper presents the analytical results obtained from implementing the impedance control method for a cantilever beam structure. Experiments were conducted to validate the open loop characteristics. The experimental procedure and the results obtained are also detailed in this paper. As a first step towards real-time implementation of the impedance controller, a dSPACE digital signal processor card is used for a simple state feedback control.

\section{IMPEDANCE CONTROL}

\subsection{Theory}

The principle of impedance control is that, for maximum power dissipation, the impedance of the controller should match the complex conjugate of the structural impedance, i.e.,

$$
\mathrm{Z}_{\mathrm{c}}=\mathrm{Z}_{\mathrm{s}}^{*}
$$

Consider a structure with impedance $Z_{s}$ that is controlled by a controller with impedance $Z_{c}$ as shown in Fig. 1 .

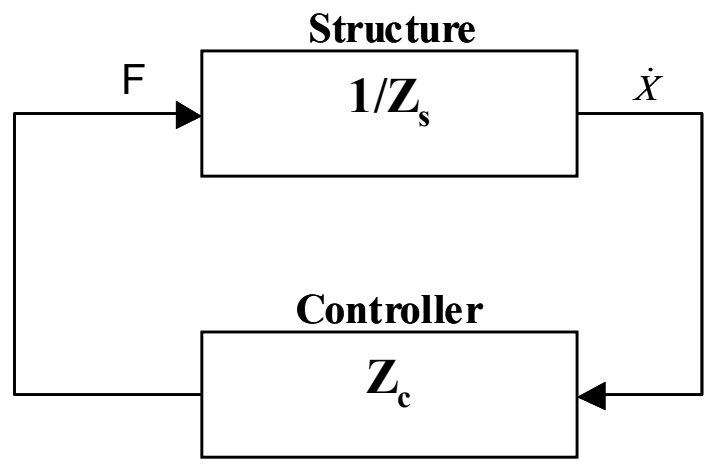

Figure 1. Block diagram for an impedance controller.

The total impedance of the structure/controller is

$$
Z_{t}=Z_{s}+Z_{c}=\left(R_{s}+R_{c}\right)+j\left(\chi_{s}+\chi_{c}\right)
$$

The power dissipated $\mathrm{P}$ in the controller is given by

$$
P=R_{c}|\dot{X}|^{2}
$$

The above equation is analogous to the power dissipated in electric circuits which is equal to the product of the resistance and the square of current flowing through it.

In eqn.(3), the velocity $\dot{X}$ is determined as,

$$
\dot{X}=F / Z_{t}
$$

Combining equations (3) and (4) gives,

$$
P=R_{c} \frac{F^{2}}{\left[\left(R_{s}+R_{c}\right)^{2}+\left(\chi_{s}+\chi_{c}\right)^{2}\right]}
$$


For a given $\mathrm{R}_{\mathrm{c}}$, the dissipated power attains maximum when $\partial \mathrm{P} / \partial \chi_{\mathrm{c}}=0$, which requires that

$$
\chi_{\mathrm{c}}=-\chi_{\mathrm{s}}
$$

Under this condition, the power dissipated is given by

$$
P_{\max }=R_{c} F /\left(R_{s}+R_{c}\right)^{2}
$$

Such a power can be maximised by selecting the resistance of the controller $R_{c}$ such that, $\partial \mathrm{P} / \partial \mathrm{R}_{\mathrm{c}}=0$, which requires that

$$
\mathrm{R}_{\mathrm{c}}=\mathrm{R}_{\mathrm{s}}
$$

Equations (6) and (8) give the optimality for maximising the power dissipated in the controller. When combined, these conditions require that $Z_{c}=R_{s}-j \chi_{s}=Z_{s}^{*}$, i.e., the impedance of the controller should match the complex conjugate of the structural impedance.

However, this controller is not realisable in practice due to its non-causal nature. An approximation to $Z_{\mathrm{s}}^{*}$ is obtained by critically damping the poles and zeros of $Z_{\mathrm{s}}{ }^{5}$. An important requirement is that the actuator-sensor pair for which the $Z_{\mathrm{s}}$ is obtained has to be a collocated pair. This can be understood as a requirement where only the local dynamics is modeled or the power flow at the actuator location is desired.

\section{EXPERIMENTS}

An experimental study was conducted with an aluminum cantilever beam to study the modal characteristics of the beam, and to compute the open loop transfer function. Two $\mathrm{ACX}^{\circledR}$ patch type piezo devices were surface mounted on the top and bottom surfaces of the beam near the fixed end. These piezo devices are each made of two piezo wafers. The technical specifications of the piezo device are given in Table 1. The schematic of the devices mounted on the beam is shown in Figure 2. These wafers are electrically isolated and hence could be used independently. The device on top of the beam is termed as P1 and the bottom device is termed as P2 and each device contains two piezo wafers, W1 and W2. Using this set of piezo devices, different actuator-sensor combinations are possible as given in the Table 2.

Table 1. Specifications of the $\mathrm{ACX}^{\circledR} \mathrm{QP} 20 \mathrm{~W}$ Piezoelectric Device

\begin{tabular}{|l|l|}
\hline Device size $(\mathrm{mm})$ & $50.8 \times 36.75 \times 0.735$ \\
\hline Device weight $(\mathrm{oz})$ & 0.28 \\
\hline Active elements & One stack of two piezos \\
\hline Device capacitance $(\mu \mathrm{F})$ & 0.20 \\
\hline Full scale voltage range $(\mathrm{V})$ & 200 \\
\hline
\end{tabular}
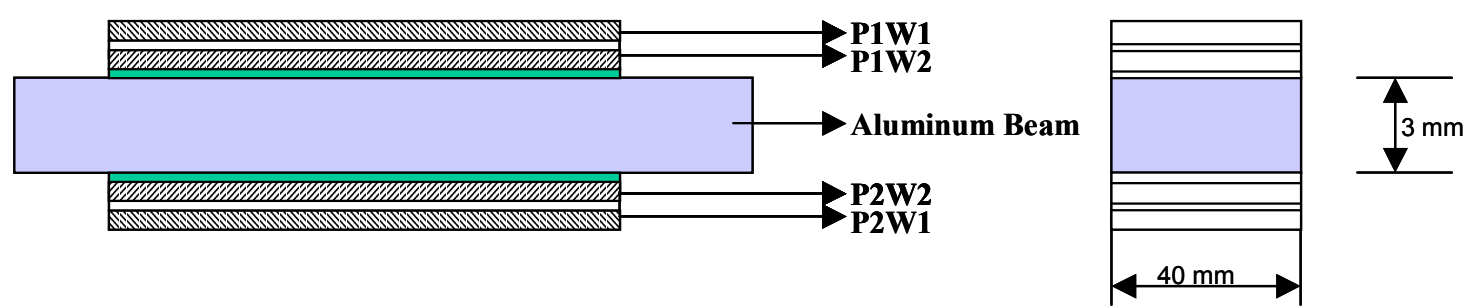

Figure 2. Schematic representation of piezo devices with beam. 
Table 2. Actuator-sensor combinations.

\begin{tabular}{|l|l|l|l|}
\hline Sl. No. & Configuration & Actuators & Sensors \\
\hline 1 & C1 & P1W1 AND P2W1 & P1W2 AND P2W2 \\
\hline 2 & C2 & P1W2 AND P2W2 & P1W1 AND P2W2 \\
\hline 3 & C3 & P1W1 AND P1W2 & P2W1AND P2W2 \\
\hline
\end{tabular}
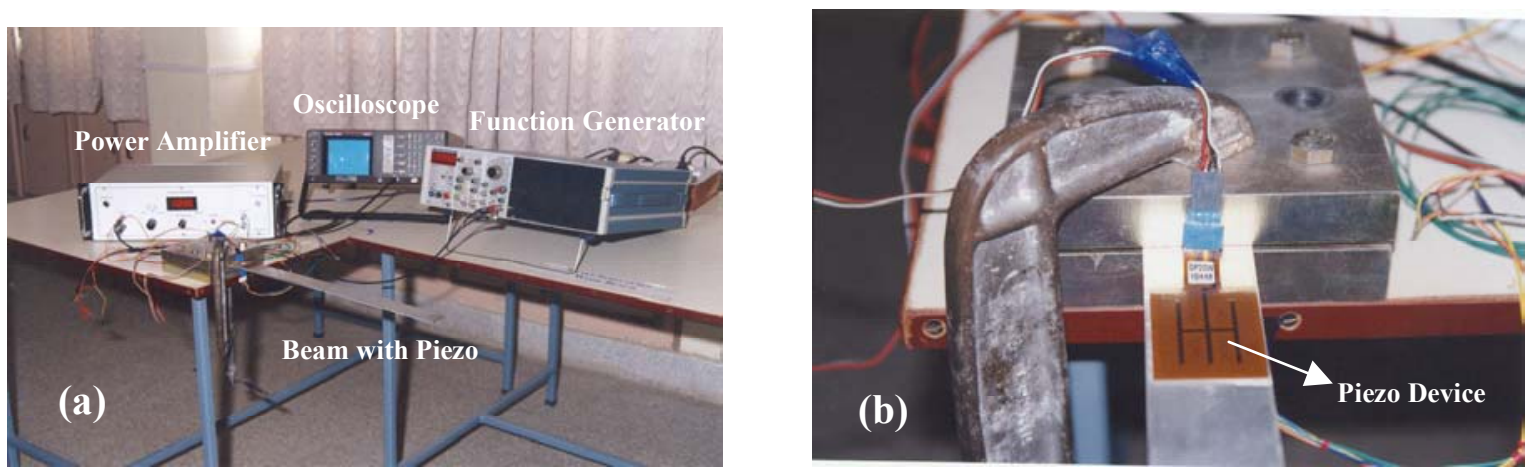

Figure 3. Determining the collocated transfer function (a) experimental setup, and (b) close-up of piezo mounted on the beam.

\subsection{Measurement of collocated transfer functions}

The experimental setup shown in Figure 3(a) is used to extract the collocated transfer function. The collocated transfer function is obtained by placing the actuator and sensor at the same location. This is done using configuration $\mathrm{C} 1$ in Table 2. The experimental transfer functions obtained for configuration $\mathrm{C} 1$ for different peak voltages applied to the actuator pairs are shown in Figure 4. The sensor output RMS voltage is directly measured at different frequencies. The peaks corresponding to the two modes can be seen in the figure. The anti-resonances expected of a collocated transfer function are also clearly observed in the figure.

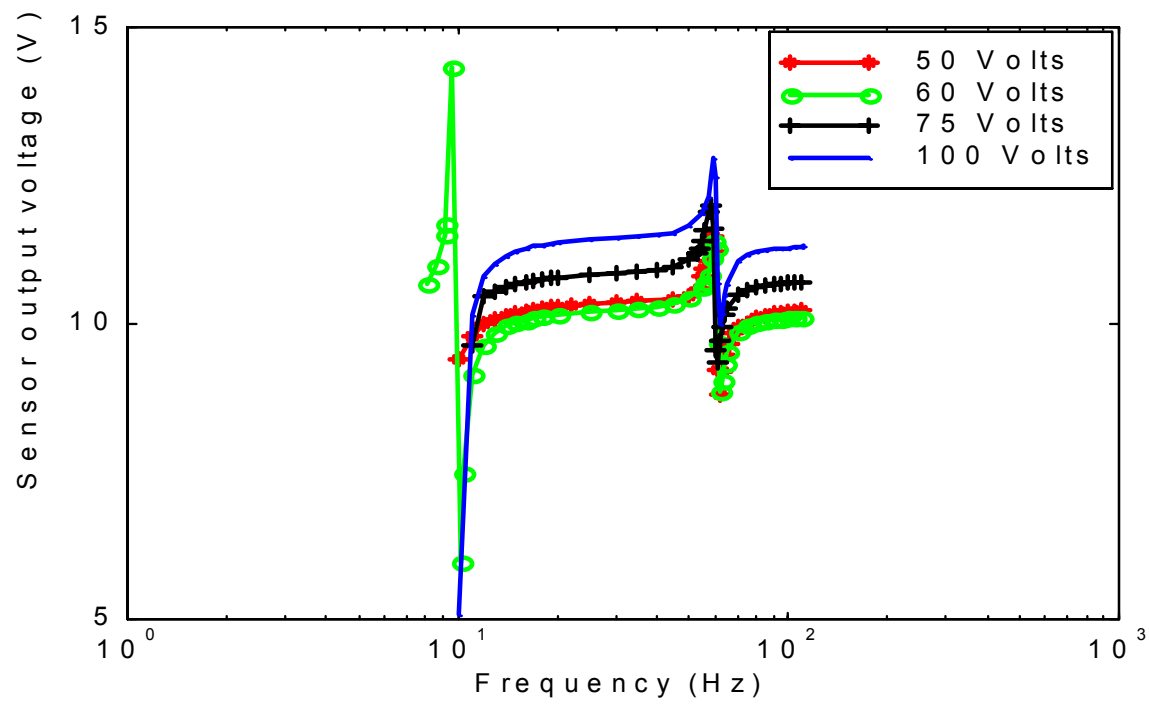

Figure 4. Experimental collocated transfer function for configuration $\mathrm{C} 1$. 


\subsection{Extraction of transfer functions using modal analysis}

Modal evaluation of transfer functions is essential where theoretical models are not available, or difficult to obtain. Mode shapes can be obtained from the experiments, which in turn are used to evaluate transfer functions of the system. Eigen vectors are obtained experimentally by fixing the sensors at specified locations of the structure. These vectors are orthonormal in nature. Since the control here aims at two prominent modes, eigen vectors corresponding to the respective modes and the damping ratios are estimated from the experiments. The mass normalised vectors can be written as

$$
\phi=\left[\begin{array}{llllllll}
\phi_{1}{ }^{1} & \phi_{2}{ }^{1} & \phi_{3}{ }^{1} & . & . & . & . & \phi_{n}{ }^{1} \\
\phi_{1}{ }^{2} & \phi_{2}{ }^{2} & \phi_{3}{ }^{2} & . & . & . & . & \phi_{n}^{2}
\end{array}\right]
$$

where, the superscript indicates the mode number, the subscript represents the modal vector element at a particular degree of freedom, and $n$ represents the number of sensors. Using the above mass normalized eigen matrix, the receptance matrix can be obtained as ${ }^{7}$

$$
[\alpha(s)]=[\phi] T\left[s^{2}+\omega_{n}^{2}\right]^{-1}[\phi]
$$

When damping is taken into consideration, the receptance matrix is given as,

$$
[\alpha(s)]=[\phi]^{T}\left[s^{2}+2 \zeta \omega_{n} s+\omega_{n}^{2}\right]^{-1}[\phi]
$$

Since the mobility is the time derivative of the receptance, the mobility matrix in the frequency domain is obtained by multiplying the receptance matrix by the Laplace variable, $s$. The procedure outlined here is a general method of obtaining the transfer function. However, in this study a finite element analysis of the beam using an Euler-Bernoulli element was done to obtain the eigen vectors and hence the mobility transfer function. Another method of obtaining the transfer function is to use curve-fitting techniques to extract the poles and zeros from the experimental data such as the one shown in Figure 4.

\section{CONTROLLER DESIGN}

After obtaining the transfer functions for the aluminum beam, the controller is obtained by critically damping the poles and zeros of the real time transfer function.

If the impedance of the controller is determined as the inverse of the structural mobility $\left(1 / Z_{\mathrm{s}}\right)$, then the problem arising from the poles-zeros cancellation remains unsolved. McMartin and Hall ${ }^{3}$ suggested the use of the direct field impedance (DFI) of the structure as an estimate of the average structural impedance. This is estimated from the reverberated structural dynamics, which is characterised by resonances (poles) and anti-resonances (zeros) resulting from the constructive or destructive interactions between the propagating through the structure and their reflections from the boundaries. The dereverberated characteristics are then determined after Betros, et $a l^{5}$.

The SIMULINK $^{\circledR}$ block diagram for the impedance control of the aluminum cantilever beam with saturation is shown in Figure 5. The saturation is introduced in the feedback loop to make the controller realistic wherein the input voltage to the actuator cannot exceed $200 \mathrm{~V}$. An initial impulse disturbance in the form of a moment is applied to the cantilever beam. The tip displacements with and without control are shown in Figure 6. 


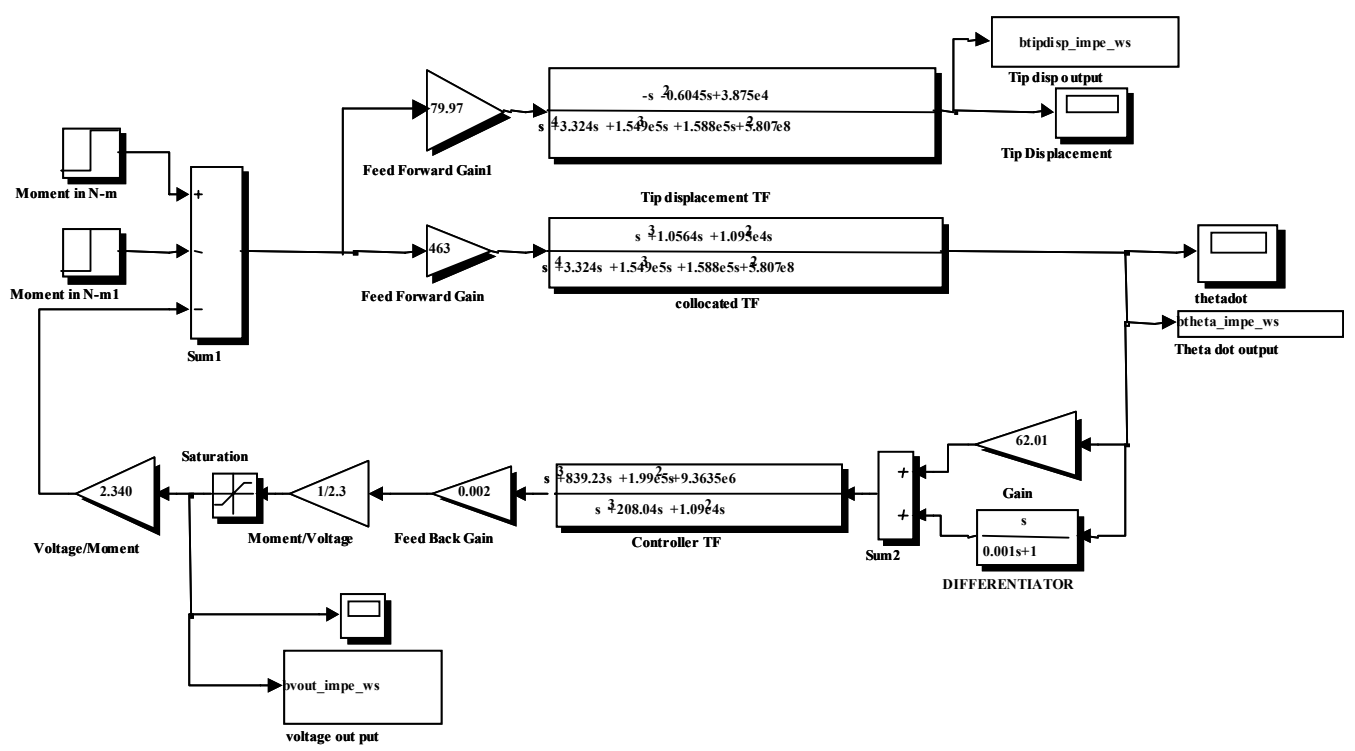

Figure 5. Simulink block diagram for the impedance control of a aluminum cantilever beam with saturation.

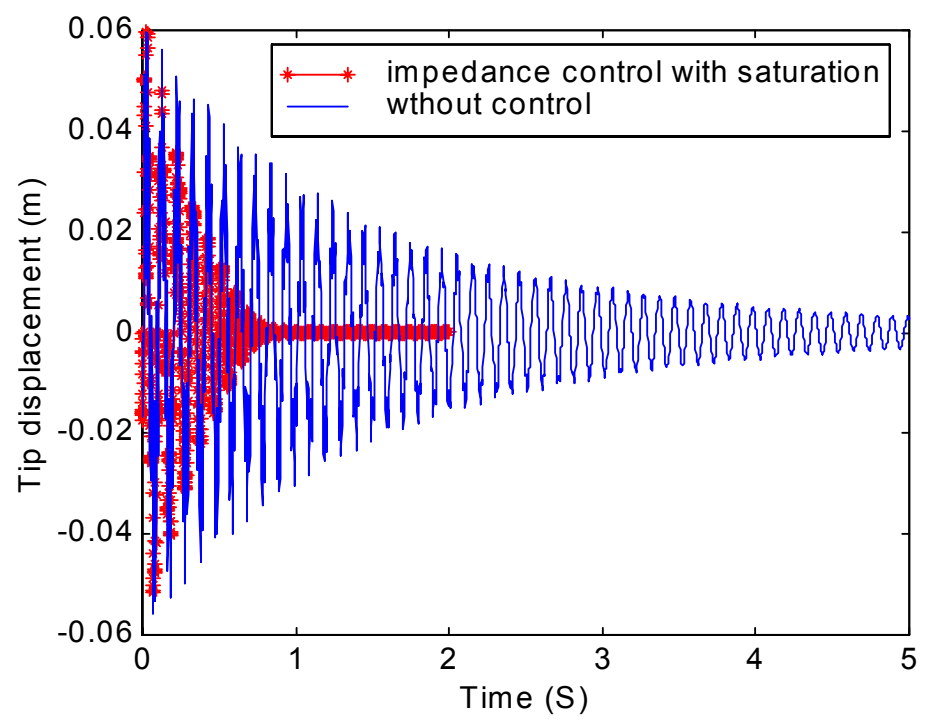

Figure 6. Tip displacement of the beam for the uncontrolled and impedance control.

\section{REAL-TIME IMPLEMENTATION OF CONTROLLER}

The real-time implementation of the controller algorithm is carried out using a dSPACE ${ }^{\circledR}$ digital signal processing (DSP) card. The card is based on a Texas Instruments TMS320C31 floating-point digital signal processor (DSP), which forms the main processing unit, providing fast instruction cycle time for numerically intensive algorithms. The board also has a set of onboard peripherals such as Analog to Digital Converters (ADC), Digital to Analog Converters (DAC) and a timer, which are frequently used in digital control systems. The 12 bit and 16 bit ADC are capable of $800 \mathrm{KHz}$ and $250 \mathrm{KHz}$ sampling speeds respectively with an input signal measurement range of $\pm 10 \mathrm{~V}$. The DSP card acquires analog signals from the piezoelectric sensor through the ADC. Raw sensor outputs are appropriately conditioned before feeding to the DSP card. The output from the controller as computed by the DSP card is amplified using a power amplifier before feeding to the piezoelectric actuator. Figure 6 shows the schematic of the real-time control system. The control algorithm is coded using MATLAB/SIMULINK ${ }^{\circledR}$ and downloaded onto the DSP card using a MATLAB-dSPACE interface. 


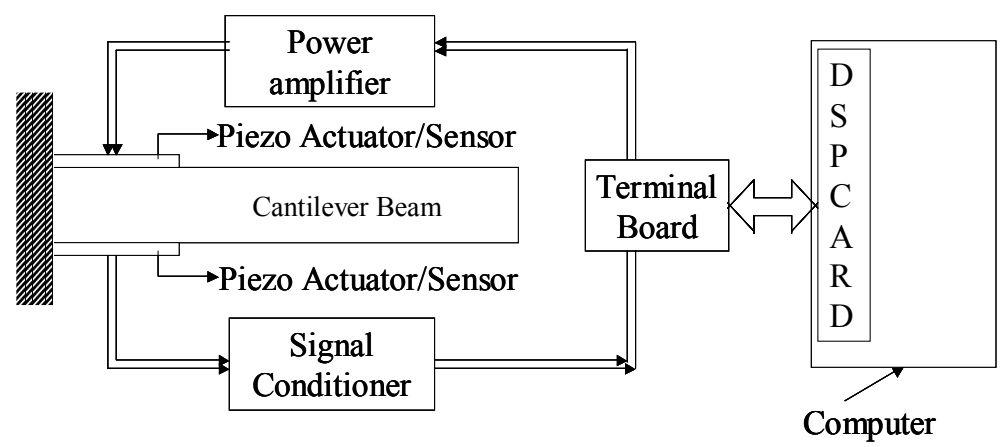

Figure 6. Schematic diagram of the real-time control system.

In order to do preliminary testing of the hardware and the software for real-time control, a simple state feedback algorithm was chosen first. A sinusoidal wave was fed as an input to the actuator through the power amplifier (with gain (G1) of 22.5) at the first resonant frequency of the cantilever beam for a period of three seconds and stopped. The beam was then left to vibrate freely. The open loop response of the cantilever beam is shown in Figure 7(a). The feedback gain is the product of the power amplifier gain (G1) and the gain set in the software (G2). The closed loop response for G2 value of -1.75 is plotted in Figure 7(b). The gain was restricted in order to prevent the feedback voltage to the actuator from exceeding the breakdown voltage. It can be seen from the plots that a reduction in the amplitude of the response of the beam is observed in the controlled case.
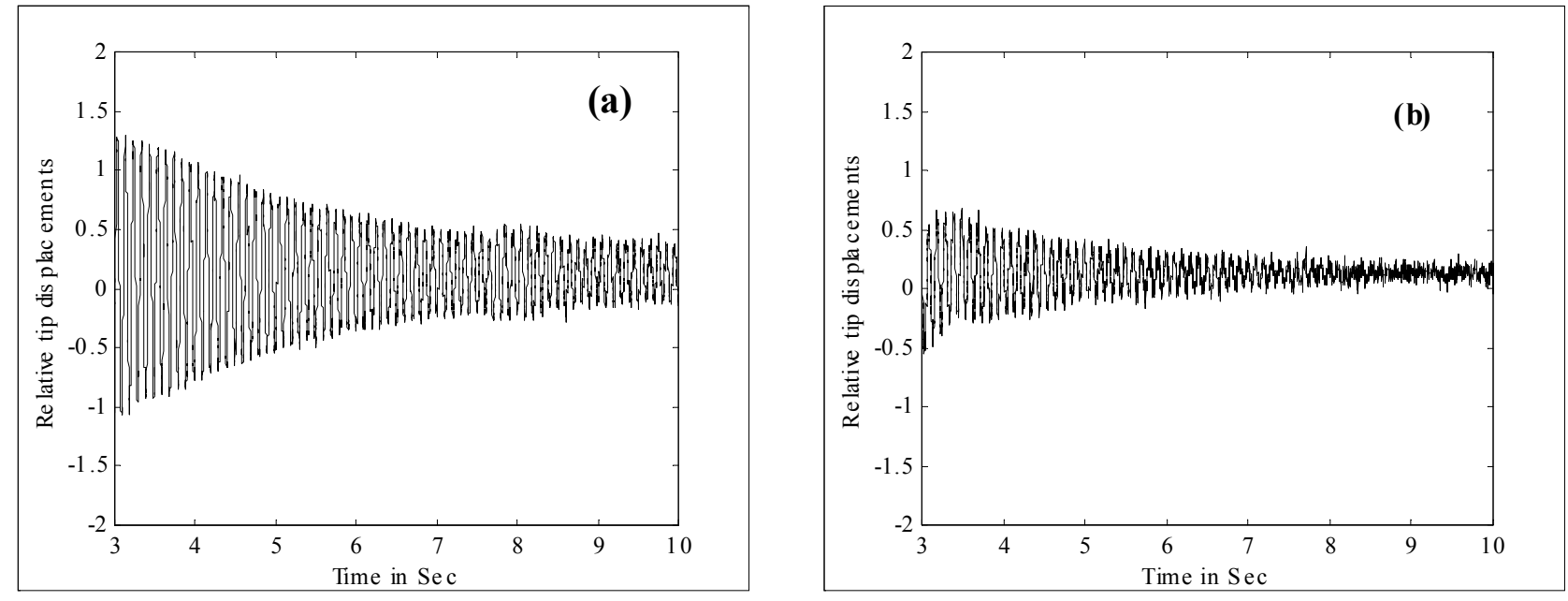

Figure 7. (a) Open loop response of cantilever beam, and (b) Closed loop response of cantilever beam.

\section{SUMMARY AND CONCLUSIONS}

An impedance control approach is investigated for the real-time control of structural vibrations. The advantage of this approach is that it can be used for realistic structures wherein the transfer functions can be obtained experimentally. A cantilever beam was chosen as a testbed for studying and implementing this methodology. Simulations were done using the impedance approach and the approach was found to be effective in reducing the vibration. This method has been compared with other conventional methods such as velocity feedback ${ }^{8}$ and independent modal space control (IMSC) ${ }^{9}$ and was found to be comparable in performance (the results of this comparison have not been presented in this paper for the sake of brevity). Preliminary testing of real-time implementation of control algorithms was conducted using a dSPACE $^{\circledR}$ DSP card with a Matlab/Simulink ${ }^{\circledR}$ interface. A simple state feedback was first attempted and found to be effective in control. Work is in progress to implement the impedance control algorithm using the DSP card. Having 
established the methodology for the cantilever beam, the next step is to study the vibration control of a more realistic structure.

\section{ACKNOWLEDGEMENTS}

The support of the Aeronautics Research and Development Board (Structures Panel) under the grant-in-aid scheme is gratefully acknowledged. The authors also thank Mr. M.Subba Rao, Head, Advanced Composites Unit for his valuable inputs during the course of this work.

\section{REFERENCES}

1. Glaese, R.M., and Miller, D.W., "A generalized impedance matching feedback law for structural-acoustic control”, Paper No. AIAA - 97 - 1209,1997.

2. Hirami, N., "An active maximum power absorber for the reduction of noise and vibration", J. Sound and Vibration, 200, No. 3, pp. $261-279,1997$

3. MacMartin, D.G., and Hall, S.R., "Control of uncertain structures using an $\mathrm{H}_{\infty}$ power flow approach", AIAA $J$. Guidance, Control and Dynamics, 14, No. 3, pp. 521 - 530,1991.

4. MacMartin, D.G., and Hall, S.R., "Structural control experiments using an $\mathrm{H}_{\infty}$ power flow approach", J. Sound and Vibration, 148, No. 2, pp. 223 - 241,1991.

5. Betros, R.S., Alvarez-Salazar, O.S., and Bronowicki, A.J., "Experiences with active damping and impedance matching compensators," Proc. SPIE 1917, Smart Structures and Intelligent Systems, pp. 856 - 869, 1993.

6. Skudrzyk, E., "The mean-value method of predicting the dynamic response of complex vibrators", J. Acoustical Society of America, 67, No. 4, pp. 1105 - 1135,1980.

7. Ewins, D.J., Modal Testing: Theory and Practice, Research Studies Press Ltd., Hertfordshire, U.K., 1986.

8. Balas, M.J., "Direct velocity feedback control of large space structures," J. Guidance and Control, 2, No. 3, pp. $252-253,1979$.

9. Meirovitch, L., Baruh, H., and Oz, H., "A comparison of control techniques for large flexible systems", AIAA $J$. Guidance, Control and Dynamics, 6, No. 4, pp. $302-310,1983$. 